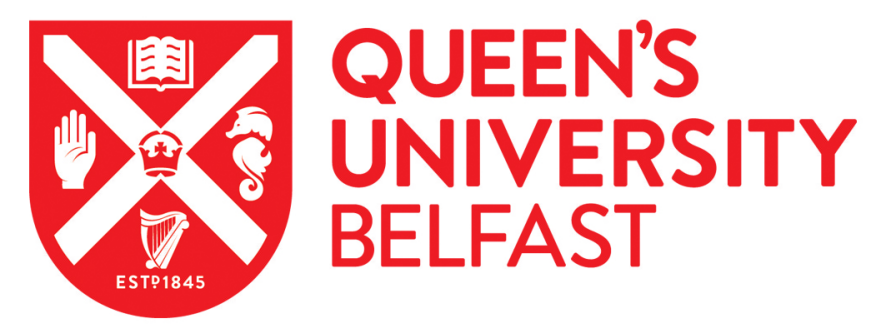

\title{
Enhanced Laser-Driven Ion Acceleration by Superponderomotive Electrons Generated from Near-Critical-Density Plasma
}

Bin, J., Yeung, M., Gong, Z., Wang, H. Y., Kreuzer, C., Zhou, M. L., Streeter, M. J. V., Foster, P., Cousens, S., Dromey, B., Meyer-ter-Vehn, J., Zepf, M., \& Schreiber, J. (2018). Enhanced Laser-Driven Ion Acceleration by Superponderomotive Electrons Generated from Near-Critical-Density Plasma. Physical Review Letters, $120,1-5$. [074801]. https://doi.org/10.1103/PhysRevLett.120.074801

Published in:

Physical Review Letters

Document Version:

Publisher's PDF, also known as Version of record

Queen's University Belfast - Research Portal:

Link to publication record in Queen's University Belfast Research Portal

Publisher rights

(C) 2018 American Physical Society. This work is made available online in accordance with the publisher's policies. Please refer to any applicable terms of use of the publisher.

\section{General rights}

Copyright for the publications made accessible via the Queen's University Belfast Research Portal is retained by the author(s) and / or other copyright owners and it is a condition of accessing these publications that users recognise and abide by the legal requirements associated with these rights.

Take down policy

The Research Portal is Queen's institutional repository that provides access to Queen's research output. Every effort has been made to ensure that content in the Research Portal does not infringe any person's rights, or applicable UK laws. If you discover content in the Research Portal that you believe breaches copyright or violates any law, please contact openaccess@qub.ac.uk. 


\title{
Enhanced Laser-Driven Ion Acceleration by Superponderomotive Electrons Generated from Near-Critical-Density Plasma
}

\author{
J. H. Bin,,${ }^{1,2}{ }^{*}$ M. Yeung, ${ }^{3}$ Z. Gong, ${ }^{4}$ H. Y. Wang, ${ }^{5,6}$ C. Kreuzer, ${ }^{1}$ M. L. Zhou, ${ }^{4}$ M. J. V. Streeter, ${ }^{7}$ P. S. Foster, ${ }^{3,8}$ \\ S. Cousens, ${ }^{3}$ B. Dromey, ${ }^{3}$ J. Meyer-ter-Vehn, ${ }^{2}$ M. Zepf,${ }^{3,5}$ and J. Schreiber ${ }^{1,2, \uparrow}$ \\ ${ }^{1}$ Fakultät für Physik, Ludwig-Maximilians-Universität München, D-85748 Garching, Germany \\ ${ }^{2}$ Max-Planck-Institut für Quantenoptik, D-85748 Garching, Germany \\ ${ }^{3}$ Department of Physics and Astronomy, Centre for Plasma Physics, Queens University Belfast, BT7 1NN Belfast, United Kingdom \\ ${ }^{4}$ State Key Laboratory of Nuclear Physics and Technology, and Key Laboratory of High Energy Density Physics Simulation, \\ CAPT, Peking University, 100871 Beijing, China \\ ${ }^{5}$ Helmholtz Institute Jena, Fröbelstieg 3, 07443 Jena, Germany \\ ${ }^{6}$ School of Environmental and Chemical Engineering, Shanghai University, 99 Shangda Road, Shanghai 200444, China \\ ${ }^{7}$ The John Adams Institute for Accelerator Science, Blackett Laboratory, Imperial College London, London SW72AZ, United Kingdom \\ ${ }^{8}$ Central Laser Facility, STFC Rutherford Appleton Laboratory, Chilton, Didcot, Oxon OX11 OQX, United Kingdom
}

(Received 25 August 2017; published 15 February 2018)

\begin{abstract}
We report on the experimental studies of laser driven ion acceleration from a double-layer target where a near-critical density target with a few-micron thickness is coated in front of a nanometer-thin diamondlike carbon foil. A significant enhancement of proton maximum energies from 12 to $30 \mathrm{MeV}$ is observed when a relativistic laser pulse impinges on the double-layer target under linear polarization. We attributed the enhanced acceleration to superponderomotive electrons that were simultaneously measured in the experiments with energies far beyond the free-electron ponderomotive limit. Our interpretation is supported by two-dimensional simulation results.
\end{abstract}

DOI: 10.1103/PhysRevLett.120.074801

The rapid development of high-power laser technology has triggered a fast evolution of laser driven particle sources. In the past two decades, ion beams generated by relativistic laser pulses interacting with solid-density targets have stimulated the idea of compact particle sources for a range of applications, and thus attract a great deal of attention [1-4]. Scaling up to higher energy and achieving a well-controlled energy spectrum has remained the key topic of current research. Investigations have been dedicated to advanced targets such as a nanometer thin foil $[5,6]$ and novel acceleration mechanisms such as the breakout afterburner (BOA) $[7,8]$ and the light sail form of radiation pressure acceleration (RPA-LS) [9-13]. These novel acceleration mechanisms promise higher ion energy, a well-controlled energy spectrum, and higher conversion efficiency but also pose new challenges for laser and target technology. For instance, RPA is a result of the accelerating field that is established when radiation pressure of the incident laser pulse pushes on plasma electrons which drag ions along. By suppression of heating the electrons when using normal incidence and circular polarization $(\mathrm{CP})$, RPA-LS has been recently demonstrated in experiments with higher beam energy and smaller energy spread [14-17].

Alternatively, it is of great interest to tackle this issue with another acceleration mechanism, the target normal sheath acceleration (TNSA) mechanism where ions are accelerated by strong electrostatic fields which are set up by laser generated hot electrons exiting the target rear. TNSA is typically the dominate acceleration mechanism in most experimental conditions. The fact that in such a scenario hot electrons are generated in the target front by absorbing laser energy, triggers enthusiastic prospects of improving ion acceleration by increasing laser absorption and conversion efficiency to hot electrons. For instance, a foambased double-layer target has been proposed by theorists [18-20] and tested in experiments recently [21]. Increased proton acceleration has been reported with targets coated with a monolayer of nanospheres [22]. In particular, superponderomotive electrons from laser-plasma interaction, which has been recently revisited both experimentally and theoretically $[23,24]$, could be a promising candidate to increase ion acceleration, which has not yet been demonstrated in experiments so far.

In this Letter, we present a novel approach to enhance ion acceleration based on a double-layer target configuration. In particular, nanometer (nm) thin diamondlike carbon (DLC) foils [5] coated with several $\mu \mathrm{m}$ thick carbon nanotube foam (CNF) targets [25] at near-critical density (NCD) are used in experiments. In contrast to our previous investigated case under circular polarization [17], the electron density of CNF employed here is estimated to be about $0.5 n_{c}$ at interacting point due to much pronounced target preexpansion under linear polarization, slightly below the critical density of electrons $n_{c}$. Significant enhancement of ion beams that 


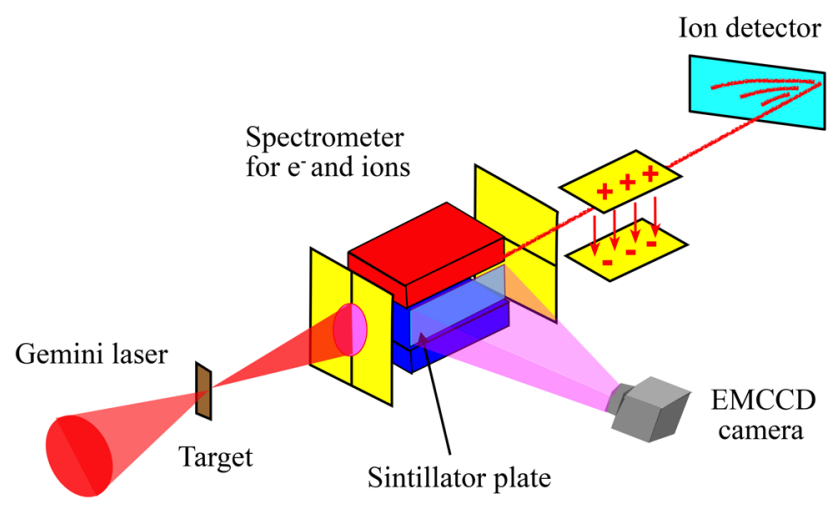

FIG. 1. Experimental setup. The Gemini laser pulse is normally incident on the target under linear polarization. A specially designed spectrometer provides simultaneous measurements of electron and ion spectrum.

accelerated from such targets as compared to pure DLC foils, has been measured in the experiments with linearly polarized incident laser pulses. The electron spectrum that was monitored in parallel suggests that the increased acceleration field can be attributed to strongly enhanced electron heating to average energies much beyond the free electron ponderomotive limit. Supporting two-dimensional (2D) particle-in-cell (PIC) simulations reveal that those superponderomotive electrons are generated from the several $\mu \mathrm{m}$ thick NCD plasma during the interaction via the direct laser acceleration (DLA) mechanism [26-28].

The experiments were performed at the Gemini laser at the Central Laser Facility of the Rutherford Appleton Laboratory in the United Kingdom. The system delivers pulses with a duration of $50 \mathrm{fs}$ full width at half maximum (FWHM) centered at $800 \mathrm{~nm}$ wavelength. The initial laser contrast is $10^{-5}$ at 2 ps before the peak of the main pulse [29]. A recollimating double plasma mirror system was introduced to enhance the laser contrast to a ratio of $10^{-9}$ at
5 ps before the peak of main pulse. 4-5 J laser energy was delivered on target.

An $f / 2$ off-axis parabolic mirror (OAP) was used to focus the laser pulses to a FWHM focal spot diameter of $3.5 \mu \mathrm{m}$, yielding a peak intensity of $2 \times 10^{20} \mathrm{~W} / \mathrm{cm}^{2}\left(a_{0} \approx 10\right)$ when considering the complete spatial distribution with high dynamic range. $20 \mathrm{~nm}$ DLC foils were coated with CNF layers with various thicknesses and were irradiated under normal incidence with linear polarization (LP).

The experimental setup is sketched in Fig. 1. In the experiments, electrons and ions were simultaneously determined with a specially designed spectrometer with moderate $b$-field strength (100-240 mT). A vertical entrance slit is located in front of the dipole magnets. Electrons were deflected by the magnetic field to a scintillator plate located inside the magnets and imaged onto an EMCCD camera. Another horizontal slit oriented perpendicular to the vertical slit was placed after the dipole magnets. Ions were measured in the target normal with those two sequential, mutually perpendicular slit entrances along with electric field plates placed after the magnets which acting as a Thomson-parabolalike setup, provided a detectable spectra range of $2-50 \mathrm{MeV} / \mathrm{u}$.

The ion results are summarized in Fig. 2 for the LP Gemini laser pulses. The energy distributions monotonically decay and terminate at a maximum energy value, both for protons and carbon ions [see Fig. 2(a)]. The energies increase with increasing CNF thickness. In particular, the maximum energy of protons increases more strongly, from 12 to $29 \mathrm{MeV}$ - a factor of 2.4-with increasing thickness of the CNF layer, while $\mathrm{C}^{6+}$ energy increases by a smaller factor of 1.7 [Fig. 2(b)]. When using freestanding CNF targets, no proton signal and only low energy carbons with maximum energy up to $\sim 3.5 \mathrm{MeV} / \mathrm{u}$ have been detected. Note that, our result is different from Ref. [30], where enhanced ion energy was observed from pure hydrogen target due to different laser and target conditions.
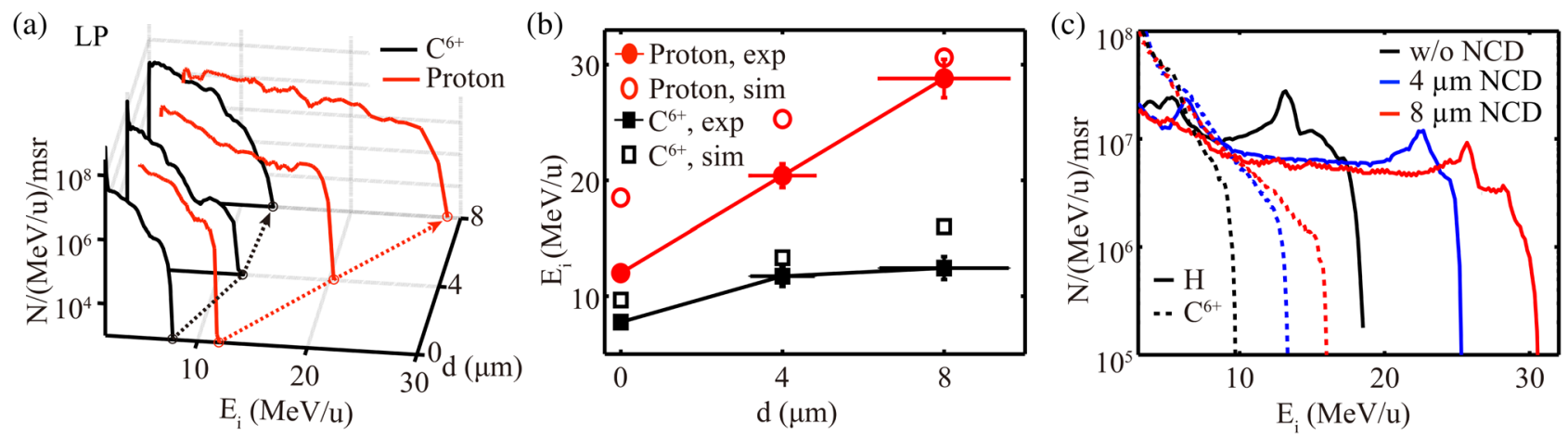

FIG. 2. (a) Ion energy distributions of protons (red) and $\mathrm{C}^{6+}$ (black) for the case of linearly polarized laser pulses interacting with $\mathrm{CNF}$ targets of varying thickness attached to a $20 \mathrm{~nm}$ DLC foil. (b) Maximum energy of proton (solid red) and carbon (solid black) beams for varying CNF thickness in double-layer target configuration. The vertical error bars denote the energy resolution of the spectrometer and the horizontal error bars present the production deviation in target thickness. (c) $\mathrm{C}^{6+}$ ion (dashed curves) and proton (solid curves) energy spectra extracted from simulations at $t=250 \mathrm{fs}$ for varying NCD thickness (color marked). The maximum energies (empty symbols) are compared with the experimental data in (b). 
Figure 3 shows the energy spectra of electrons measured simultaneously to the corresponding ion spectra in Fig. 2. The spectrum measured from a pure $20 \mathrm{~nm}$ DLC foil (without CNF) presents a typical quasithermal distribution (black curve). An exponential fit to the measured electron energy distribution yields an electron temperature $T_{h}$ of $3.8 \mathrm{MeV}$, comparable to the ponderomotive temperature $T_{h, \text { pond }}=\left(\sqrt{1+a_{0}^{2} / 2}-1\right) m_{e} c^{2}[31]$ for $a_{0}=10$.

With an additional CNF layer attached to the DLC foil, more pronounced electron heating is observed. Both the temperature $T_{h}$ and the total energy, which is represented by $n_{h} T_{h}$, of the hot electrons increase with CNF thickness. The most energetic electron spectrum was obtained with an $8 \mu \mathrm{m}$-thick CNF layer, yielding superponderomotive electrons with $T_{h}$ of about $8 \mathrm{MeV}$, much higher than the free-electron ponderomotive scaling at identical intensity $\left(T_{h}=3.6 \mathrm{MeV}\right)$, and the total electron energy $n_{e} T_{h}$ is enhanced by a factor of 6.2 as compared to a single layer DLC foil (see the inset in Fig. 3). The vertical error bars denote the difference between estimated $T_{h}$ to $\bar{E}-E_{\min }$, where mean electron energy $\bar{E}$ is deduced within the resolved spectral range starting from optional minimum energy $E_{\min }=10 \mathrm{MeV}$. In other words, they present the deviation from the measured spectral to an ideal exponential curve. The horizontal error bars represent the production deviation in target thickness.

The strong correlation between the spectra of electrons and ions suggests the important role of those superponderomotive electrons in the acceleration process and in fact is consistent with a simple physical picture. The ions are accelerated in an electric field set up by fast laseraccelerated electrons, the accelerating field is thus

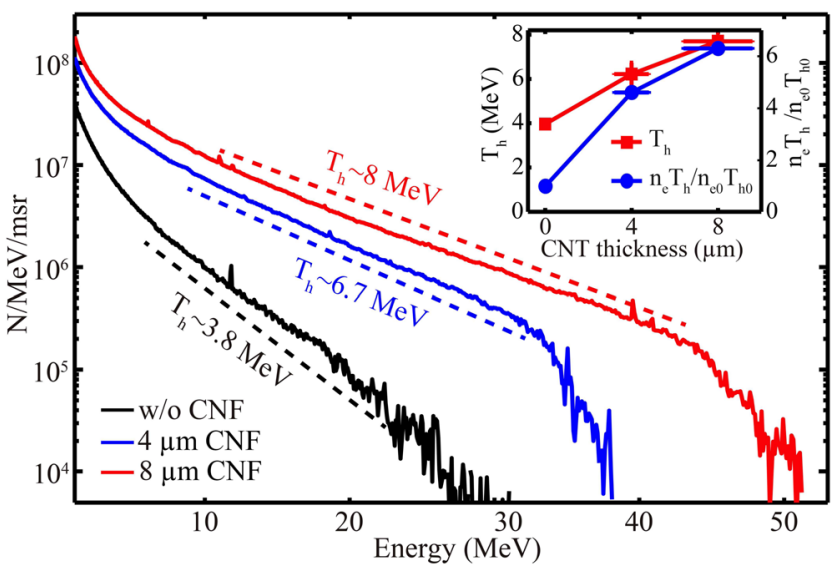

FIG. 3. Measured electron energy spectra along target normal (laser propagating direction) with LP pulses interacting with CNF targets with different thickness attached to a $20 \mathrm{~nm}$ DLC foil. The inset shows the comparison of corresponding electron temperature $T_{h}$, and total electron energy as presented by the production $n_{e} T_{h}$ after normalization to the value $n_{e 0} T_{h 0}$ from $20 \mathrm{~nm}$ DLC foil solely. Both quantities $n_{e}$ and $T_{h}$ are extracted from the fitting curve. determined by $E_{\text {acc }} \propto \sqrt{n_{e} T_{h}}$ regardless of the actual dynamics [32,33]. One would then expect the resultant ion energy scale to be $\sqrt{n_{e} T_{h}}$. Such a hypothesis is in reasonable agreement with our experimental results where we found that the optimal enhancement $(2.4 \times)$ is consistent with the measured enhancement in total energy of superponderomotive electrons $n_{e} T_{h}(6.2 \times)$. The reduced energy gain of carbon $(1.7 \times)$ indicates that they experience the same acceleration field integral but with only half the charge to mass ratio compared to protons. Off course, in practice the exact dependence might be complicated by details of the dynamics. Nevertheless, the observed superponderomotive electrons are expected to enhance laser-driven ion acceleration as a general trend.

To further understand the interaction, 2D PIC simulations were carried out with EPOCH code [34]. A solid density $\left(80 \mathrm{~nm}, n_{0}=150 n_{c}\right.$, where $n_{c}$ is the critical density) plasma slab was used to present the employed DLC foils $\left(20 \mathrm{~nm}, 600 n_{c}\right)$. A NCD plasma slab $\left(0.5 n_{c}\right)$ with different lengths represents the CNF targets. The initial temperature of the electrons is $1 \mathrm{keV}$. The simulation box is $100 \lambda$ in the laser direction $(Z)$ and $30 \lambda$ in the transverse direction $(Y)$ in 2D with a resolution of 100 cells $/ \lambda$ and 25 cells $/ \lambda$, respectively. Each cell is filled with 28 macroparticles. A linearly polarized laser pulse, with peaked intensity $I_{0}=2 \times 10^{20} \mathrm{~W} / \mathrm{cm}^{2}$, with a Gaussian envelope in both the spatial and temporal distribution with a FWHM diameter $D_{L}$ of $3.5 \mu \mathrm{m}$ and a FWHM duration of $50 \mathrm{fs}$, is used to approximate the experiment conditions.

Figure 4(a) shows the quantitative energy distribution of electrons obtained from double-layer targets with different NCD plasma lengths. We observe a significant increase with increasing NCD plasma length, agreeing well with our experimental results. In particular, the superponderomotive behavior is reproduced with thicker NCD plasma. The simulation result indicates an efficient DLA process. When the laser propagates through the NCD plasma, it forms a well-defined plasma channel confining most of the laser energy [upper graph of Fig. 4(b)]. The electrons oscillate at betatron frequency when comoving with the laser along the channel. When the oscillating frequency resonates with the laser frequency [26,35], the energy of the laser field is then transferred to electrons via the DLA process along the plasma channel in the NCD plasma [30,36,37]. Such resonance behavior is clearly seen in the lower graph of Fig. 4(b), where the electron energy density distribution closely resembles the laser intensity distribution and oscillates at twice the laser cycles. Consequently, the electrons gradually gain energy from the laser pulse when it propagates through the NCD plasma. This finally leads to superponderomotive electrons with both higher electron temperature and electron number, as shown in Fig. 4(c). In fact, these DLA electrons constitute a major part of the total electron energy distribution, as indicated by the green 

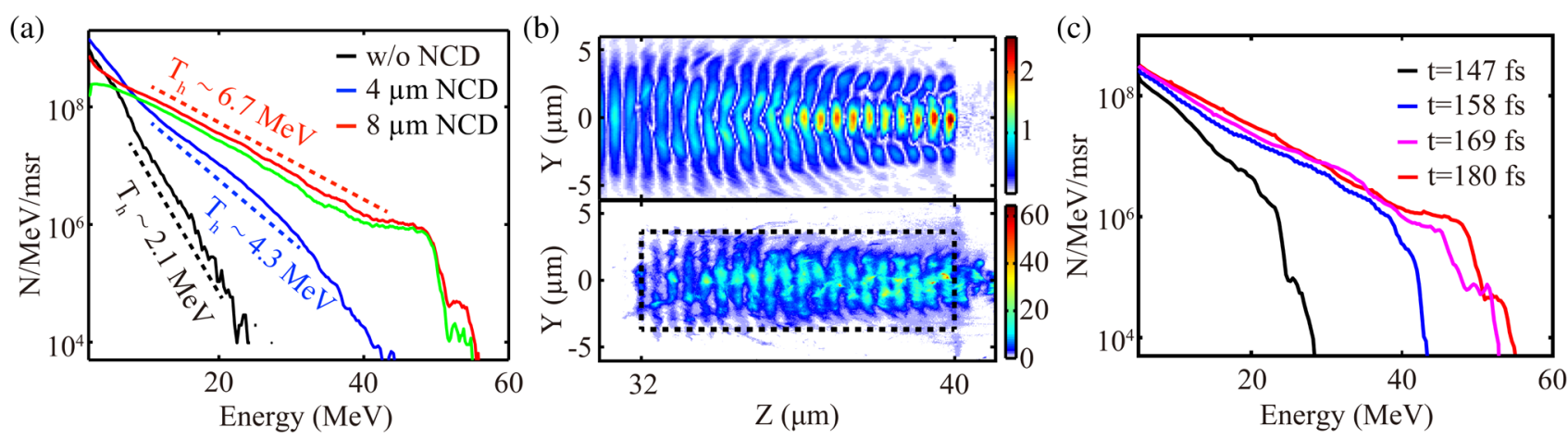

FIG. 4. 2D PIC simulation results. (a) Absolute electron energy distribution with different NCD plasma length, the time is chosen when the electron cutoff energy reaches a maximum in individual cases. The green curve shows the electron spectrum from the area identified by the black dashed box in (b). (b) Simulated absolute value of laser field distribution normalized by initial $a_{0}$ (upper) and energy density of the electrons from NCD plasma normalized by $n_{c} m_{e} c^{2}$ (lower) at $t=180$ fs, for the $8 \mu$ m case in (a). Here the NCD plasma is located in the range of 32-40 $\mu \mathrm{m}$, whereas the solid density target is placed between $40 \mu \mathrm{m}$ and $40.08 \mu \mathrm{m}$. The black dashed box marks the DLA electrons from NCD plasma within laser focal volume. (c) Temporal evolution of electron energy distribution for the $8 \mu \mathrm{m}$ case. The peak performance coincides at the time $(t=180 \mathrm{fs})$ when the laser peak approaches the front surface of the solid density target.

curve in Fig. 4(a) which is extracted from the marked area in the black dashed box.

Note that relativistic self-focusing [38] can benefit the generation of superponderomotive electrons [20]. While, as we only employed NCD targets with much lower density, the target thickness in our case is far below the optimum length for a relativistic self-focusing effect. For example, one would expect a self-focusing length of about $16 \mu \mathrm{m}$ with a NCD target at density of $0.5 n_{c}$ [17]. Therefore, we judge the impact from relativistic self-focusing under our condition to be small. It could be further explored in future experiments.

As expected, the superponderomotive electrons give rise to enhanced ion acceleration, as evidenced in Fig. 2(c). In agreement with the experiments, we observed strong enhancement of both proton and carbon ion energies with increasing NCD plasma length. In general, the energy distributions for both species exhibit a monotonically decaying shape, similar to experimental observations. More importantly, the observed energy per nucleon of protons are by a factor of $\sqrt{2}$ larger than carbon ions, a feature that is expected from the plasma expansion mechanism $[32,33]$. Further support of our hypothesis is shown in Fig. 2(b), where the maximum energies were extracted from simulations and plotted versus the NCD thickness, showing fair agreement with the experimental results.

In conclusion, we have demonstrated the positive effect of superponderomotive electrons for laser driven ion acceleration. Realized by double-layer target configuration, we generated superponderomotive electrons from a first NCD density layer which drive consecutive ion acceleration from attached nanometer thin foils. The significant enhancement of proton energies by a factor of 2.4 could represent a new path towards high efficient ion acceleration and is of great importance to approaching relevant applications that require higher kinetic energies with a much released power requirement for the laser technology.

This work was supported by the DFG-funded Cluster of Excellence Munich Centre for Advanced Photonics (MAP). The experiment was funded by UK Engineering and Physical Sciences Research Council (EPSRC) and the A-SAIL and LIBRA grants. We thank the staff of the Gemini operations team and the Central Laser Facility for assistance during the experiment. H. Y.W. acknowledges financial support from National Natural Science Foundation of China under Grant No. 11605111. We also acknowledge essential target support from W. J. Ma. The work is also partly funded by UK EPSRC Grants No. EP/ L02327X/1 and No. EP/P016960/1. The PIC code EPOCH was funded by the UK EPSRC Grants No. EP/G054950/1, No. EP/G056803/1, No. EP/G055165/1, and No. EP/ M022463/1.

*Present address: Lawrence Berkeley National Laboratory, Berkeley, California 94720, USA.

jianhuibin@lbl.gov

Joerg.Schreiber@lmu.de

[1] M. Borghesi, J. Fuchs, S. Bulanov, A. Mackinnon, P. Patel, and M. Roth, Fusion Sci. Technol. 49, 412 (2006).

[2] H. Daido, M. Nishiuchi, and A. S. Pirozhkov, Rep. Prog. Phys. 75, 056401 (2012).

[3] A. Macchi, M. Borghesi, and M. Passoni, Rev. Mod. Phys. 85, 751 (2013).

[4] J. Schreiber, P. R. Bolton, and K. Parodi, Rev. Sci. Instrum. 87, 071101 (2016).

[5] W. Ma, V. Liechtenstein, J. Szerypo, D. Jung, P. Hilz, B. Hegelich, H. Maier, J. Schreiber, and D. Habs, Nucl. Instrum. Methods Phys. Res., Sect. A 655, 53 (2011).

[6] J. H. Bin et al., Phys. Plasmas 20, 073113 (2013). 
[7] L. Yin, B. J. Albright, B. M. Hegelich, and J. C. Fernández, Laser Part. Beams 24, 291 (2006).

[8] L. Yin, B. J. Albright, B. M. Hegelich, K. J. Bowers, K. A. Flippo, T. J. T. Kwan, and J. C. Fernández, Phys. Plasmas 14, 056706 (2007).

[9] A. Macchi, F. Cattani, T. V. Liseykina, and F. Cornolti, Phys. Rev. Lett. 94, 165003 (2005).

[10] O. Klimo, J. Psikal, J. Limpouch, and V. T. Tikhonchuk, Phys. Rev. ST Accel. Beams 11, 031301 (2008).

[11] X. Q. Yan, C. Lin, Z. M. Sheng, Z. Y. Guo, B. C. Liu, Y. R. Lu, J. X. Fang, and J. E. Chen, Phys. Rev. Lett. 100, 135003 (2008).

[12] A. P. L. Robinson, M. Zepf, S. Kar, R. G. Evans, and C. Bellei, New J. Phys. 10, 013021 (2008).

[13] B. Qiao, M. Zepf, M. Borghesi, and M. Geissler, Phys. Rev. Lett. 102, 145002 (2009).

[14] A. Henig et al., Phys. Rev. Lett. 103, 245003 (2009).

[15] S. Kar et al., Phys. Rev. Lett. 109, 185006 (2012).

[16] S. Steinke et al., Phys. Rev. ST Accel. Beams 16, 011303 (2013).

[17] J. H. Bin et al., Phys. Rev. Lett. 115, 064801 (2015).

[18] T. Nakamura, M. Tampo, R. Kodama, S. V. Bulanov, and M. Kando, Phys. Plasmas 17, 113107 (2010).

[19] A. Sgattoni, P. Londrillo, A. Macchi, and M. Passoni, Phys. Rev. E 85, 036405 (2012).

[20] H. Y. Wang, X. Q. Yan, J. E. Chen, X. T. He, W. J. Ma, J. H. Bin, J. Schreiber, T. Tajima, and D. Habs, Phys. Plasmas 20, 013101 (2013).

[21] I. Prencipe et al., Plasma Phys. Controlled Fusion 58, 034019 (2016).
[22] D. Margarone et al., Phys. Rev. Lett. 109, 234801 (2012).

[23] S. Jiang et al., Phys. Rev. Lett. 116, 085002 (2016).

[24] A. Sorokovikova, A. V. Arefiev, C. McGuffey, B. Qiao, A. P. L. Robinson, M. S. Wei, H. S. McLean, and F. N. Beg, Phys. Rev. Lett. 116, 155001 (2016).

[25] W. Ma et al., Nano Lett. 7, 2307 (2007).

[26] A. Pukhov, Z.-M. Sheng, and J. M. ter Vehn, Phys. Plasmas 6, 2847 (1999).

[27] C. Gahn, G. D. Tsakiris, A. Pukhov, J. Meyer-ter-Vehn, G. Pretzler, P. Thirolf, D. Habs, and K. J. Witte, Phys. Rev. Lett. 83, 4772 (1999).

[28] S. P. D. Mangles et al., Phys. Rev. Lett. 94, 245001 (2005).

[29] C. Hooker, Y. Tang, O. Chekhlov, J. Collier, E. Divall, K. Ertel, S. Hawkes, B. Parry, and P. P. Rajeev, Opt. Express 19, 2193 (2011).

[30] M. H. Helle, D. F. Gordon, D. Kaganovich, Y. Chen, J. P. Palastro, and A. Ting, Phys. Rev. Lett. 117, 165001 (2016).

[31] W. L. Kruer and K. Estabrook, Phys. Fluids 28, 430 (1985).

[32] P. Mora, Phys. Rev. Lett. 90, 185002 (2003).

[33] J. Schreiber et al., Phys. Rev. Lett. 97, 045005 (2006).

[34] T. D. Arber et al., Plasma Phys. Controlled Fusion 57, 113001 (2015).

[35] B. Liu, H. Y. Wang, J. Liu, L. B. Fu, Y. J. Xu, X. Q. Yan, and X. T. He, Phys. Rev. Lett. 110, 045002 (2013).

[36] L. Willingale et al., IEEE Trans. Plasma Sci. 36, 1825 (2008).

[37] S. S. Bulanov et al., Phys. Plasmas 17, 043105 (2010).

[38] M. Borghesi, A. J. MacKinnon, L. Barringer, R. Gaillard, L. A. Gizzi, C. Meyer, O. Willi, A. Pukhov, and J. Meyer-terVehn, Phys. Rev. Lett. 78, 879 (1997). 